\title{
Depósito de objetos de aprendizagem em repositórios a partir da integração com ambientes virtuais de aprendizagem
}

\author{
Marcelo Augusto Rauh Schmitt, IFRS \\ marcelo.schmitt@poa.ifrs.edu.br \\ Liane Margarida Rockenbach Tarouco, CINTED-UFRGS \\ liane@penta.ufrgs.br \\ Alessandra Pereira Rodrigues, IFSul \\ ale@pelotas.ifsul.edu.br \\ Jonathan Alba Videira, IFRS \\ jonathan.videira@gmail.com
}

Resumo. A fim de compartilhar o que é produzido por diferentes equipes e permitir a reutilização de materiais pedagógicos que promovam a aprendizagem, a utilização de repositórios de objetos de aprendizagem torna-se essencial. Por outro lado, cada vez mais docentes fazem uso de ambientes virtuais de aprendizagem para disponibilização de cursos aos alunos. Neste contexto em que se apresentam duas ferramentas distintas, a duplicidade de interfaces pode ser um fator de desmotivação para que professores publiquem objetos de aprendizagem em repositórios abertos que comumente disponibilizam em ambientes virtuais de aprendizagem. $O$ presente artigo tem como objetivo apresentar uma solução que integra repositórios de objetos de aprendizagem implementados sobre o software DSpace e o ambiente virtual de aprendizagem MOODLE através do padrão SWORD. O plugin desenvolvido permite que a unificação das interfaces e a diminuição do número de passos necessários para a publicação de objetos de aprendizagem nos dois ambientes.

Palavras-chave: Repositório de objetos de aprendizagem, objeto de aprendizagem, ambiente virtual de aprendizagem.

Abstract. To share what is produced by different teams and allow reuse of learning materials that promote learning, the use of repositories of learning objects is essential. Moreover, teachers are increasingly using virtual environments learning courses available for students. In this context we present two different tools, the dual interfaces can be a demotivating factor for teachers to publish learning objects in open repositories of learning objects that provide commonly in virtual learning environments. This article aims to present a solution that integrates learning object repositories implemented on the DSpace software and the Moodle virtual learning environment through the standard SWORD. The plugin developed enables the unification of interfaces and reducing the number of steps required for the publication of learning objects in both environments.

Keywords: repository of learning objects, learning object, a virtual learning environment 


\section{Introdução}

Ao longo dos anos, o uso das tecnologias da informação e comunicação aplicadas a educação vem intensificando-se nas escolas e universidades. Diversos recursos educacionais são desenvolvidos nas mais diferentes instituições: textos, hiperdocumentos, vídeos, simulações, animações, até mesmo mundos virtuais. $\mathrm{O}$ desenvolvimento de conteúdo educacional demanda recursos econômicos e humanos que necessitam ser bem administrados. Por esta razão, é fundamental que aquilo que é produzido por tantas instituições de ensino possa ser disponibilizado para o maior conjunto possível de profissionais da educação. A disponibilização de recursos educacionais digitais pode trazer benefícios para a melhoria do ensino e evitar a duplicação de esforços na sua produção.

Objetos de aprendizagem (OA) constituem um paradigma que busca viabilizar a reutilização de recursos educacionais digitais nos mais diversos cenários de aprendizagem. De acordo com Wiley (2000), "um objeto de aprendizagem é qualquer recurso digital que possa ser reutilizado para suportar a aprendizagem". Segundo Audino e Nascimento (2010, p. 141), “objetos de aprendizagem são recursos digitais dinâmicos, interativos e reutilizáveis em diferentes ambientes de aprendizagem elaborados a partir de uma base tecnológica". Um OA pode ser um vídeo, imagem, áudio, texto, gráfico, tabela, tutorial, aplicação, mapa, jogo educacional, animação, infográfico, página $W e b$, etc. Os OAs cobrem diversas modalidades de ensino, com diversos campos de atuação e possuem características como durabilidade, facilidade para atualização, flexibilidade, interoperabilidade, modularidade, portabilidade, entre outras (Audino e Nascimento, 2010, p. 141). Os OAs também podem ser facilmente manipulados e agregados a outros OAs ou qualquer outra mídia digital. Um OA pode ter usos variados, como também seu conteúdo ou layout podem ser alterados a fim de melhor se adaptarem a cada situação. Ao utilizar um OA já existente que melhor se adapte às suas necessidades, o profissional deixa de dispensar tempo e produtividade, sendo que esse tempo ganho pode ser utilizado para outros fins, como para o planejamento das aulas

Todavia, a simples criação de objetos de aprendizagem segundo parâmetros de reusabilidade não garante a sua reutilização. É preciso que os objetos de aprendizagem sejam facilmente localizáveis. O professor deve ser capaz de encontrar os objetos úteis ou necessários às suas atividades pedagógicas. Poder-se-ia considerar a possibilidade de disponibilização em sites da Internet. Desta forma, os recursos poderiam ser buscados por ferramentas como o Google. Essa solução mostra-se inadequada uma vez que a pesquisa por OAs precisa ser indexada a partir de informações pedagogicamente úteis (tipo de objeto, público-alvo, duração, licença de uso, nível de interatividade). Outra alternativa seria a colocação dos objetos de aprendizagem em Ambientes Virtuais de Aprendizagem (AVA). Essa também não se constitui em opção viável na medida em que o acesso a esse tipo de ambiente é, via de regra, restrito aos membros de uma instituição. Há dois requisitos que devem ser satisfeitos por quaisquer mecanismos de busca de objetos de aprendizagem: metadados padronizados e visibilidade externa. $\mathrm{O}$ sistema que implementa essas funcionalidades é conhecido como Repositório de Objetos de Aprendizagem (ROA).

Grande parte das instituições de ensino pelo mundo utilizam ambientes virtuais de aprendizagem como tecnologia básica para disponibilização de objetos de aprendizagem aos alunos. A utilização de interfaces únicas em sistemas é fator de aumento de produtividade e de conforto para o usuário. Com a utilização de ROAs e AVAs, o professor depara-se com uma variedade de interfaces e uma redundância de 
tarefas. Tarouco, Rodrigues e Schmitt (2013) demonstram como o processo de disponibilização em um AVA de um objeto de aprendizagem que já está em um ROA é pouco natural. Problema semelhante ocorre quando um professor deseja enviar um OA para um repositório ao mesmo tempo em que pretende disponibilizá-lo para os seus alunos no AVA. Neste caso, há necessidade de primeiro adicionar o OA ao repositório e, posteriormente, adicionar a referência no AVA. Acredita-se que essa duplicação de etapas para publicação de OAs é um dos fatores que desmotiva o professor e que impede que a promessa da reusabilidade seja finalmente cumprida.

Sob o aspecto da seleção e inclusão de OAs em AVAs a partir de repositório digital, Rodrigues (2013) desenvolveu um plugin para a integração destes ambientes através do protocolo de comunicação SRU/W - Search and Retrieval via URL e Search/Retrieve Web Service (SRU, 2007). Decorrentes da testagem do plugin desenvolvido com alunos e professores foram conhecidos benefícios para ambos os usuários, como: a integração realizada apoiou professores na construção de um plano de ensino e aprendizagem na medida em que permitiu e facilitou a pesquisa e a seleção de materiais educacionais digitais adequados, facilitou o acesso a materiais educacionais confiáveis e permitiu a reutilização desses materiais através do repositório utilizado. No caso dos alunos, a integração realizada apoiou a adequação do plano proposto pelo professor às suas necessidades individuais, na medida em que foi possível localizar e utilizar os OAs adequados às necessidades disponíveis no repositório e que os OAs foram considerados importantes na aprendizagem pelos alunos, visto que dirimiram dúvidas que outros materiais não haviam sido suficientes para o entendimento do conteúdo. Outro resultado encontrado foi a motivação os alunos em realizarem pesquisas com maior frequência que a de costume, reduzindo as pesquisas no momento que antecede a avaliação formal e distribuindo os estudos ao longo do tempo, decorrente da facilidade de pesquisar e encontrar materiais confiáveis para estudo.

Este artigo apresenta uma proposta de integração entre um Ambiente Virtual de Aprendizagem e um Repositório de Objetos de Aprendizagem sob o aspecto da publicação de Objetos de Aprendizagem, através de padrão de interconexão SWORD (Simple Web-service Offering Repository Deposit) (SWORD, 2012). SWORD é um padrão de interoperabilidade que permite aos repositórios digitais aceitarem o depósito de conteúdo de várias fontes em diferentes formatos, através de um protocolo padronizado. Da mesma forma que o protocolo HTTP permite que qualquer navegador web possa conversar com qualquer servidor web, assim o protocolo SWORD permite aos clientes falar com os servidores de repositório. SWORD foi definido com vistas a oferecer uma solução para facilitar o envio de recursos para sistemas repositórios acadêmicos.

A fim de se analisar a viabilidade da proposta, foi realizada a implementação desta solução para interligar o AVA MOODLE ao ROA DSpace, permitindo que o professor possa adicionar novos objetos ao repositório através da interface do Ambiente Virtual de Aprendizagem.

\section{Repositórios de Objetos de Aprendizagem}

Um repositório de objetos de aprendizagem, segundo Silveira, Omar, e Mustaro (2007, p. 132), é definido como um catálogo digital, com a finalidade de facilitar a pesquisa por OAs. Tais sistemas permitem o armazenamento propriamente dito, o controle de versões e de publicação, a busca a partir de suas características, o controle de acesso e a 
avaliação dos objetos. A principal diferença entre ROAs e outros repositórios digitais está no gênero de objetos armazenados: objetos de aprendizagem. Tal característica remete à ideia de que recursos educacionais armazenados em ROAs devem ser livres e abertos. O movimento dos Recursos Educacionais Abertos (REA) surgiu com o objetivo de promover o compartilhamento livre de conteúdo (OECD, 2007). Esse movimento segue três diretrizes fundamentais: o acesso ao conteúdo aberto deve ser gratuito, o conteúdo deve ser licenciado a fim de permitir a reutilização e a modificação e os sistemas e ferramentas utilizados devem disponibilizar o código-fonte (OLCOS, 2006).

Para possibilitar que esses OAs sejam usados e reutilizados em diferentes contextos e ambientes de aprendizagem, algumas características devem ser analisadas, tais como: granularidade, interoperabilidade e reusabilidade. Para que seja garantido a interoperabilidade de tais OAs, é recomendado que os mesmos utilizem padrões reconhecidos como LOM (LOM, 2010), IMS (IMS, 2011), SCORM (ADL, 2011), Dublin Core (DCMI, 2011), entre outros. Esses padrões utilizam metadados que têm o objetivo de permitir a busca por um OA apropriado para cada situação de aprendizagem que o professor deseja construir. O padrão LOM deu origem a grande parte dos padrões citados acima, mas cada padrão possui suas particularidades. O padrão Dublin Core foi o primeiro a ser disseminado em sistema de recuperação de conteúdo multimídia e caracteriza-se por uma maior simplicidade (menor quantidade de metadados) do que o padrão LOM.

Segundo Tarouco, Rodrigues e Schmitt (2013), grande parte dos repositórios digitais possui características de CMSs (Content Management Systems). Um CMS é uma aplicação $W e b$ que fornece serviços para criação e edição de páginas $W e b$. A grande vantagem dos CMSs é que permitem que usuários com pouco conhecimento na área de tecnologia de informação possam gerenciar suas páginas. Assim, programas como Joomla, Mambo, Drupal, eGroupware (Stylite, 2012), MrCute2 (Kilcoyne, 2012), DSpace (Dspace, 2012) permitem que seus administradores construam páginas a partir de modelos e ferramentas disponibilizados. As principais características presentes em um CMS são: definição e alteração do layout através de modelos (templates), organização do conteúdo de forma hierárquica, definição de estruturas de menus, definição de papéis de usuários relacionados com o fluxo de publicação (leitor, autor, revisor, editor, administrador), adição de ferramentas de terceiros (fóruns, chats, repositórios de imagens, repositórios de arquivos) e controle de fluxo de trabalho (workflow) (Rodrigues et al., 2009).

Segundo Fertalj et al. (2009), embora os AVAs permitam a exportação de conteúdo para outras aplicações, a comunicação com repositórios externos não é padronizada. Tal padronização é fundamental para que um Ambiente Virtual de Aprendizagem possa interagir com um algum Repositório de Objetos de Aprendizagem. Os principais protocolos de comunicação utilizados por repositórios são: SRU/W, OAIPMH, SWORD, RSS e ATOM (Paihama, 2009). São de especial interesse para este trabalho os protocolos SRU/W e SWORD, já que possibilitam a comunicação padronizada com Repositórios de Objetos de Aprendizagem com os objetivos de recuperação e inserção de objetos. 


\section{Modelo proposto para publicação de objetos de aprendizagem}

Considerando-se a situação de um professor que utilize um AVA para gerenciar o processo de ensino e aprendizagem e disponibilize nesse ambiente objetos de aprendizagem, ele precisa enfrentar dois tipos de problemas:

a) multiplicidade de interfaces quando precisa acessar mais de um ambiente para realizar sua atividade docente;

b) repetição de tarefas de publicação para disponibilizar os materiais educacionais digitais (objetos de aprendizagem) aos seus alunos.

O ambiente cotidiano para o professor é o AVA. Este é o ambiente frequentado para interagir com os alunos, para enviar arquivos, para disponibilizar um curso. $\mathrm{O}$ acréscimo de mais uma interface constitui-se em mais um elemento a tornar mais complexa sua atividade. A fim de publicar um objeto de aprendizagem tanto no AVA quanto no ROA, o professor usualmente depara-se com os seguintes passos:

1. Acessar o ROA

2. Adicionar o objeto ao ROA

3. Anotar a referência para o objeto no ROA

4. Acessar o AVA

5. Adicionar a referência anotada ao AVA

O desejável seria adicionar o objeto de aprendizagem a ambos ambientes em uma única ação. O mesmo problema de excesso de ações foi constatado por Tarouco, Rodrigues e Schmitt (2013) para a situação em que o professor necessita buscar um objeto de aprendizagem em um repositório e disponibilizá-lo em um Ambiente Virtual de Aprendizagem. Propõe-se, neste caso, um mecanismo que permita utilizar a interface do Ambiente Virtual de Aprendizagem como único ponto de acesso do usuário para realizar a publicação de objetos de aprendizagem em repositórios. A mesma interface utilizada para disponibilização de recursos didáticos dentro do AVA é utilizada para a disponibilização no ROA, eliminando a necessidade de aprendizado de nova interface e diminuindo o número de passos necessários para realização da ação. A $\quad$ Figura 1 apresenta o modelo proposto e implementado.

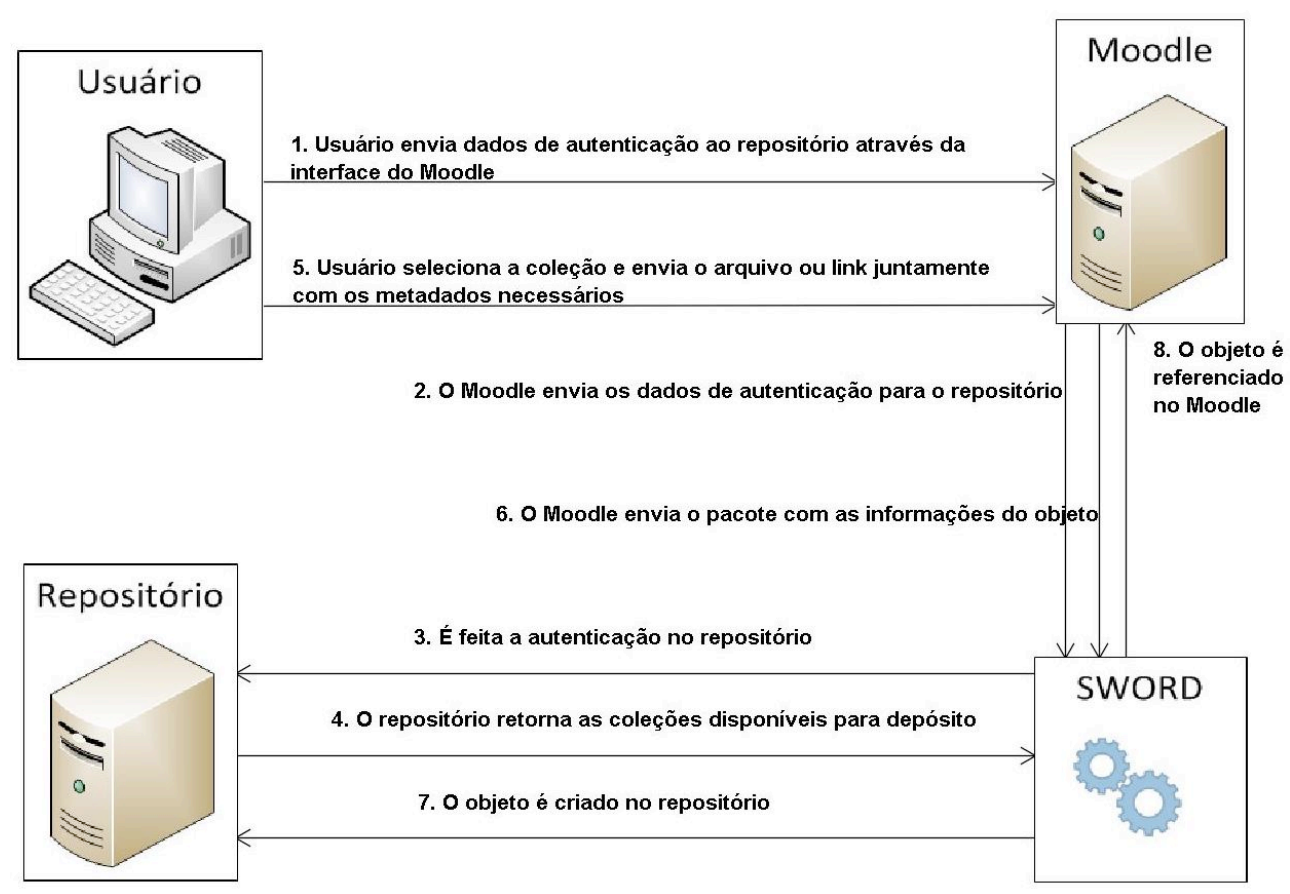

Figura 1. Etapas do modelo proposto. Fonte própria. 
O usuário comunica-se apenas com o AVA e este, através do módulo de compatibilidade desenvolvido, comunica-se com o ROA pelo protocolo SWORD.

Ao analisar o modelo, é possível verificar que o usuário realizará duas ações: acesso ao AVA e publicação objeto no AVA. O envio do objeto para o ROA, bem como a sincronização com o AVA será realizada pelo sistema.

\section{Implementação do modelo proposto}

O modelo proposto foi implementado utilizando-se um AVA e um ROA de domínio público: MOODLE e DSpace, respectivamente. Foi desenvolvido um software que realiza a integração entre as duas ferramentas através do protocolo SWORD.

O DSpace é um software de código fonte aberto desenvolvido em Java que fornece os serviços e ferramentas para a administração de um ROA. Aceita uma grande variedade de formatos de OAs como textos, imagens, vídeo, áudio, simulações, etc. O DSpace está licenciado sob a BSD Open Source License (Opensource, 2012). Para poder se adaptar a realidade e às necessidades de cada instituição, o DSpace permite (Dspace, 2012]:

- Personalização da interface ou utilização de temas prontos para que o repositório se assemelhe com o site da instituição. Existem duas formas de customização: a interface tradicional (baseada em JSP) e a utilização do framework Manakin (Dspace, 2012), baseado em XML, o qual fornece temas prontos de terceiros;

- Personalização dos metadados referentes aos OAs. O Dublin Core é o formato padrão de metadados do DSpace, mas é possível adicionar ou remover qualquer campo para ajustá-lo a suas necessidades, tal como foi realizado no repositório CESTA2 (Tarouco; Schmitt, 2010);

- Utilização de diversos protocolos padronizados, tais como OAI-PMH (Oai, 2012), OAI-ORE, SWORD [Sword, 2012], WebDAV, OpenSearch, OpenURL, RSS e ATOM;

- Utilização de mecanismos de autenticação local, como LDAP, Shibboleth, X.509 e baseado em IP. O DSpace também possui um método interno de autenticação e permite a customização de um plug-in de autenticação própria, caso possua um mecanismo de autenticação personalizado;

- Escolha de banco de dados entre PostgreSQL ou Oracle para a gerência de seus metadados;

- Escolha do idioma da aplicação. O DSpace está disponível em mais de 20 idiomas.

O AVA Moodle foi selecionado por seu amplo e disseminado uso pela comunidade acadêmica (Moodle, 2013). Ele possui uma arquitetura específica para o gerenciamento de repositórios, permitindo o desenvolvimento de códigos chamados plug-ins para a integração com outros repositórios não presentes em sua instalação.

Neste trabalho, foi desenvolvido um plugin para a inserção de objetos nos repositórios cadastrados na interface de administração (sword_upload, diponível em http://github.com). Neste caso, o professor inicialmente autentica-se no repositório no qual possui autorização de publicação a partir do AVA (Figura 2); a seguir, descreve o 
objeto preenchendo os campos correspondentes aos metadados (Figura 3); e, por último, escolhe o arquivo ou o link que corresponde ao objeto de aprendizagem a ser publicado. Ao passar essas informações para o plugin, o objeto é criado no repositório e também é adicionado ao ambiente virtual. A adição ao Ambiente Virtual de Aprendizagem pode ocorrer através de uma referência ao repositório ou através da duplicação do arquivo do próprio AVA.

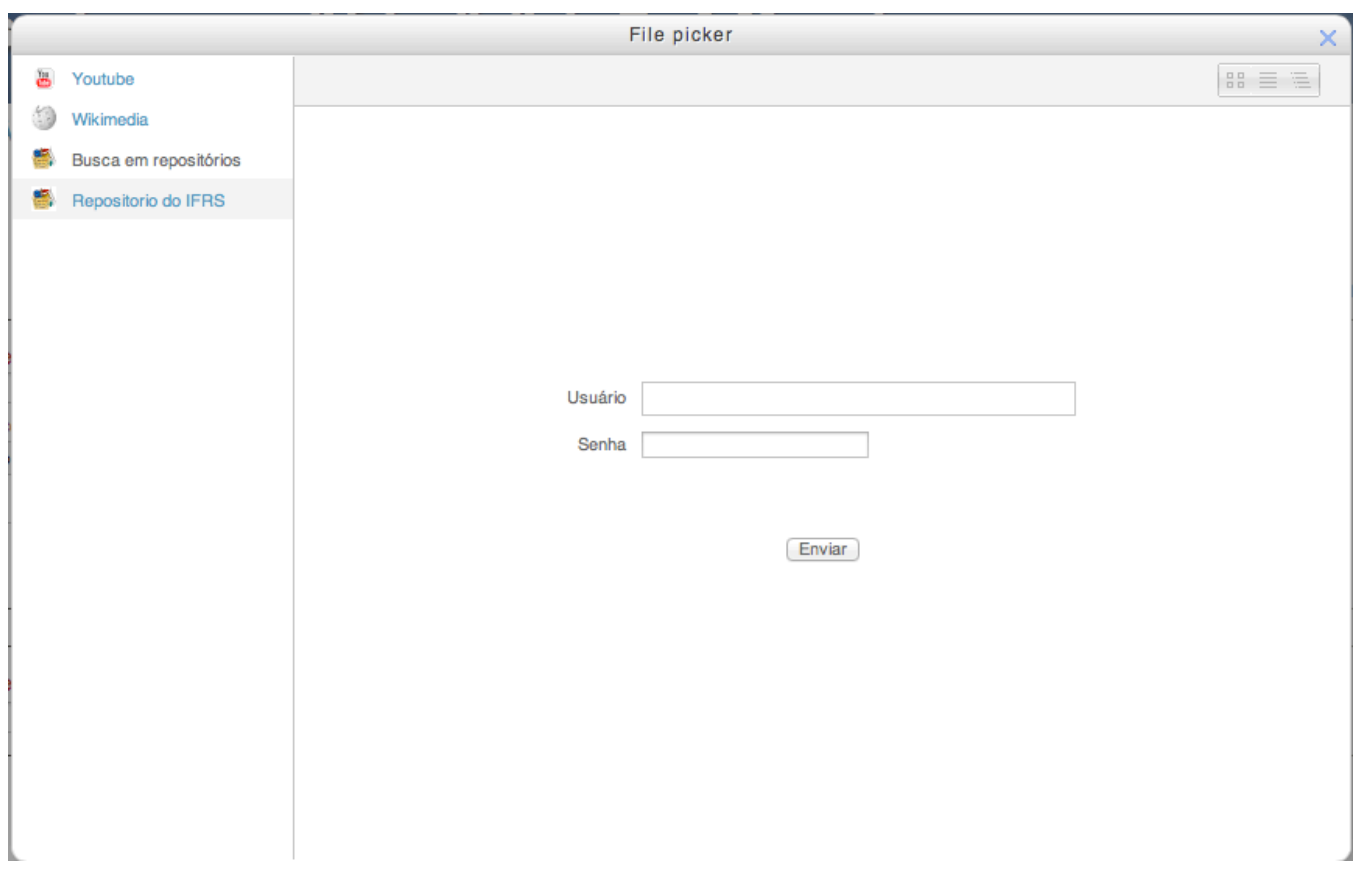

Figura 2. Interface de inserção de objetos - autenticação no ROA a partir do AVA. Fonte própria.

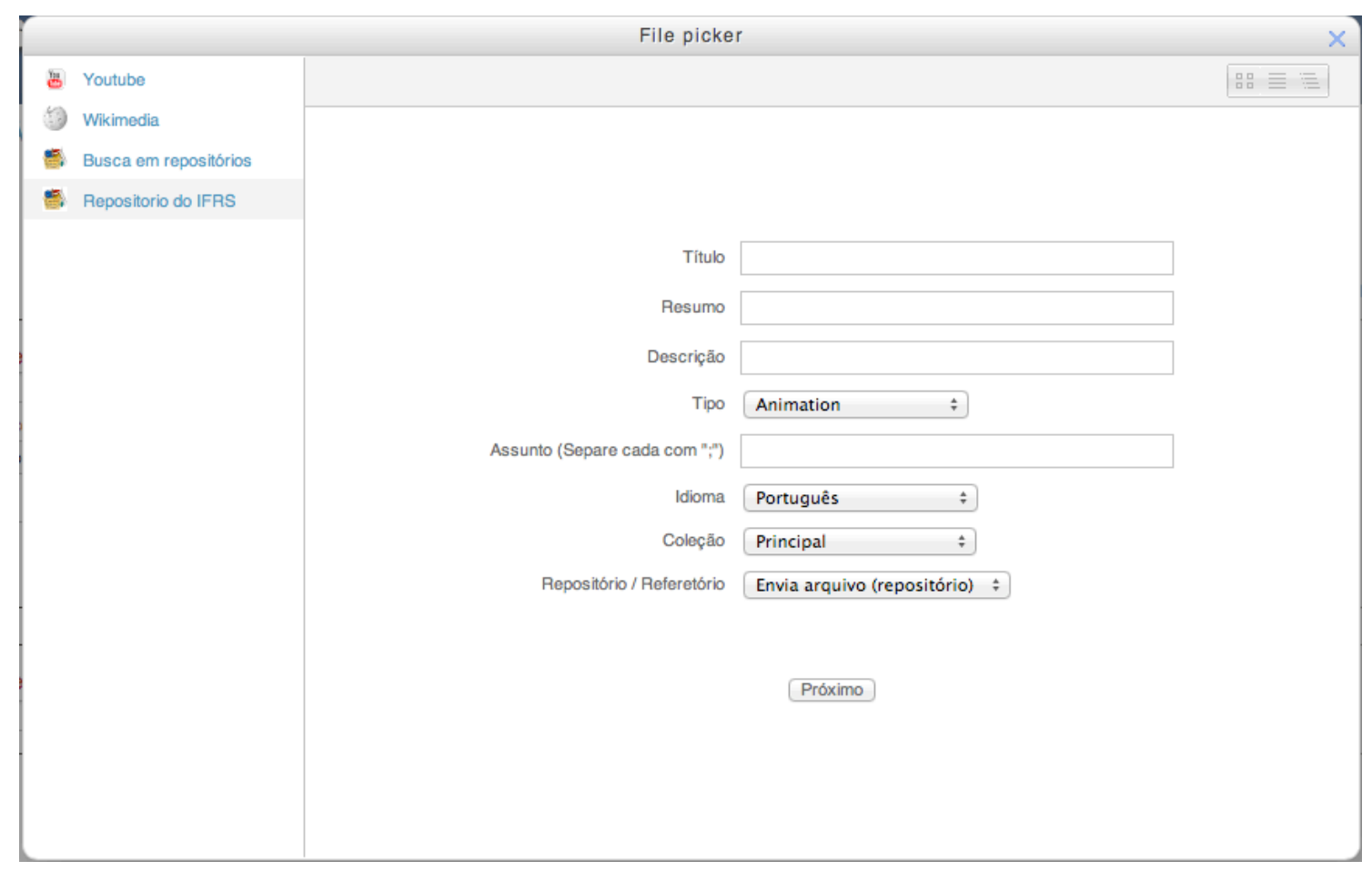

Figura 3. Interface de inserção de objetos - preenchimento de metadados. Fonte própria. 
Para a devida integração entre o AVA Moodle e os repositórios, foi necessário utilizar um padrão de comunicação presente na arquitetura dos repositórios. Para a inserção de objetos, foi utilizado o protocolo SWORD, pois ele facilita o processo de depósito de recursos e também pelo fato desse protocolo preencher os principais requisitos do ambiente de integração MOODLE-repositório (implementação do cliente em PHP e utilização do protocolo HTTP para transferência de dados).

SWORD (Sword, 2012) significa Simple Web-service Offering Repository Deposit (depósito em repositório como uma oferta da Web simples). Esse protocolo foi projetado para facilitar o depósito de recursos em sistemas como repositórios. A utilização de um padrão de interoperabilidade alivia a carga de desenvolvimento de clientes para depositar tais recursos (Dlib, 2013). A comunicação utilizando o SWORD ocorre em duas etapas. A primeira etapa requer que o usuário realize a sua autenticação no servidor (repositório). Na segunda etapa é realizado o depósito do objeto de aprendizagem propriamente dito. A figura 4 apresenta um esquema dessas duas etapas.

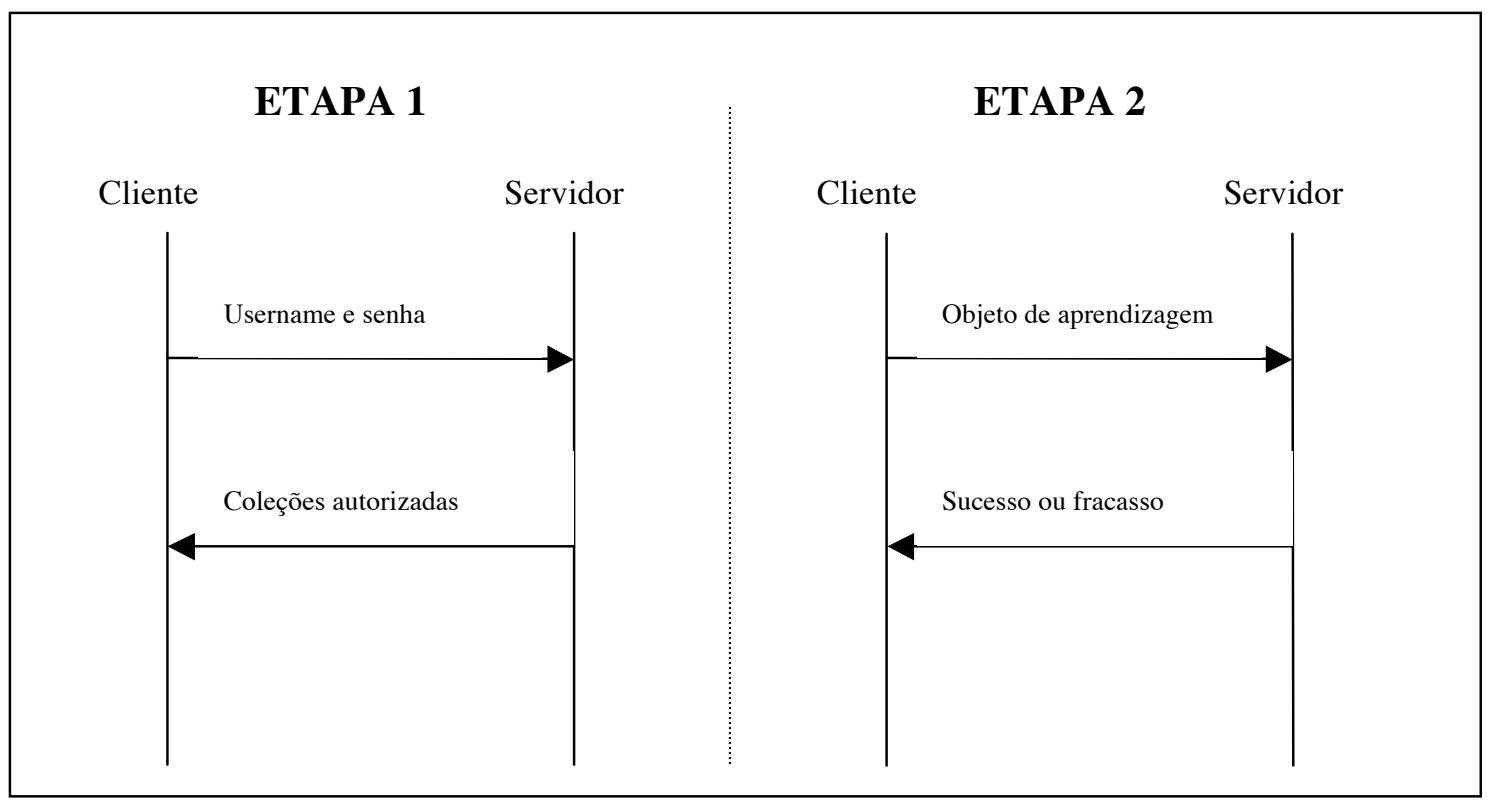

Figura 4 - Fases do protocolo SWORD como utilizado no software desenvolvido.

Para a primeira etapa finalizar sem problemas, o usuário deve fornecer um username e uma senha existentes no repositório e que tenha direitos de publicar em alguma coleção. Uma vez realizada a autenticação, o objeto de aprendizagem pode ser enviado ao repositório, na segunda etapa, juntamente com seus metadados. Esse objeto será aceito de acordo com a configuração do servidor (tamanho, tipo, metadados).

\section{Conclusões}

O desenvolvimento de um plugin que integrasse o Ambiente Virtual de Aprendizagem ao repositório de objetos de aprendizagem mostrou-se adequado na medida em que o professor não necessita de tantas etapas para disponibilizar seus materiais em repositórios e utiliza uma única interface. $\mathrm{O}$ modelo implementado proposto não se 
limita à utilização com as ferramentas MOODLE e DSpace. Qualquer repositório de objetos de aprendizagem que tenha a capacidade de se comunicar através do protocolo SWORD poderá ser integrado. Assim como desenvolveu-se um módulo para o MOODLE, é possível desenvolver-se módulos semelhantes para outros Ambientes Virtuais de Aprendizagem.

Rodrigues (2013) comprovou benefícios para alunos e professores na utilização de um repositório digital integrado a um AVA, a partir do plugin de integração entre ROA e AVA que permitiu a seleção de OAs disponíveis no repositório através do AVA, plugin este que deu origem a este trabalho. Contudo, outros estudos de caso devem ser realizados viabilizando a testagem dessa nova funcionalidade do plugin, buscando uma constatação efetiva de que a integração permite a publicação de OAs em repositórios e AVAs simultaneamente e que essa funcionalidade pode promover uma maior utilização dos repositórios de objetos de aprendizagem e trazer benefícios aos seus usuários.

\section{Referências}

ADL. Advanced Distributed Learning. Disponível em: <http://www.adlnet.org $>$. Acesso em: 18 out. 2012.

AUDINO, D.; NASCIMENTO, R. (2010). Objetos de aprendizagem - diálogos entre conceitos e uma nova proposição aplicada à educação. Revista Contemporânea de Educação, $\quad$ v. $\quad 5, \quad$ p. 128-148. Disponível em $<$ http://www.educacao.ufrj.br/artigos/n10/objetos_de_aprendizagem.pdf $>$. Acesso em: 27 dez. 2012.

DCMI. Dublin Core Metadata Initiative. Disponível em: $<$ http://dublincore.org>. Acesso em: 18 out. 2012.

DLIB. SWORD: Facilitating Deposit Scenarios. Disponível em $<$ http://www.dlib.org/dlib/january12/lewis/01lewis.html>. Acesso em 14 fev. 2013

DSPACE. Disponível em: <http://www.dspace.org>. Acesso em 13 set. 2012.

FERTALJ. K; HOIC-BOZVIC, N. \& JERKOVIC, H. (2009) Analysis of E-Learning Repository Systems and Frameworks with Prepositions for Improvements. In: Proc. Of the ITI $200931^{\text {st }}$ Int: Conf. on Information Technology Interfaces.

IMS. Instructional Management Systems. Disponível em: <http://www.imsglobal.org>. Acesso em: 18 out. 2012.

KILLCOYNE, P. MrCute2. Worcester College of Technology. Disponível em: $<$ http://www.learningobjectivity.com/mrcute>. Acesso em: 22 out. 2012.

LOM. Learning Object Metadata. Disponível em: $<$ http://1tsc.ieee.org/wg12>. Acesso em: 18 out. 2012.

MOODLE. Estatísticas Moodle. Disponível em $<\mathrm{http}: / /$ moodle.org/stats $>$. Acesso em 18 jun. 2013.

MUSTARO, P. N.; SILVEIRA, I. F.; OMAR, N; STUMP, S. M. D. Structure of Storyboard for Interactive Learning Objects Development. In: alex Koohang; Keith Harman. (Org.). Learning Objects and Instructional Design. 1 ed. Santa Rosa, California: Informing Science Press, 2007, v. 3, p. 253-280.

OAI. Disponível em <http://www.openarchives.org/pmh/>. Acesso em: 18 nov. 2012. 
OECD, 2007. Giving knowledge for free: The emergence of open educational resources. Disponível em <http://www.oecd.org/dataoecd/35/7/38654317.pdf $>$. Acesso em: 16 out. 2012.

OLCOS. Open eLearning Content Observatory Services. 2006. Disponível em http://www.olcos.org/. Acesso em Julho de 2013.

OPENSOURCE. BSD - Open Source Initiative. Disponível em: $<$ http://opensource.org/licenses/bsd-license.php>. Acesso em: 7 nov. 2012.

PAIHAMA, Jorgina. Suleman, Hussein. A Survey of the Effectiveness of Current Interoperability Protocols Technical Report. 2009. Disponível http://pubs.cs.uct.ac.za/archive/00000530/01/Interoperability_Survey_Results_Techn ical_Report.pdf. Acesso em julho de 2013

RODRIGUES, Alessandra Pereira. Integração de ambiente virtual de aprendizagem com repositório digital. 2013. 188 f. Tese (Doutorado) - Programa de Pósgraduação em Informática na Educação, PGIE, UFRGS, Porto Alegre, 2013. Disponível em: <http://www.lume.ufrgs.br/handle/10183/70617>. Acesso em: 02 set. 2013.

RODRIGUES, A. P.; SCHMITT, M. A. R.; TAROUCO, L. M. R.; VICCARI, R. M. (2009) Gestão colaborativa de conteúdo educacional. RENOTE. Revista Novas Tecnologias na Educação, v. 7, p. 13975. Disponível em $<$ http://seer.ufrgs.br/renote/article/view/13975>. Acesso em: 29 out. 2012.

SRU. Search/Retrieval via URL. 2007. Disponível em: $<$ http://www.loc.gov/standards/sru>. Acesso em 13 dez. 2012.

STYLITE AG. eGroupware. Disponível em: <http://www.egroupware.org>. Acesso em: 22 out. 2012.

SWORD. Simple Web-service Offering Repository Deposit. 2012. Disponível em $<$ http://swordapp.org $>$. Acesso em 15 dez. 2012.

TAROUCO, L. M. R. ; RODRIGUES, A. P. ; SCHMITT, M. A. R. Integração do MOODLE com Repositórios Abertos. PERSPECT CIENC INF, v. 18, p. 66-85, 2013.

TAROUCO, L. M. R.; SCHMITT, M. A. R. Adaptação de metadados para repositórios de objetos de aprendizagem. RENOTE: Revista de Novas Tecnologias na Educação, Porto Alegre, v. 8, n. 2, jul. 2010.OLCOS. Open Educational Practices and Resources. Disponível em $<$ http://www.olcos.org/cms/upload/docs/olcos_roadmap.pdf $>$. Acesso em 22 jul. 2013.

WILEY, D. A. Connecting learning objects to instructional design theory: A definition, a metaphor, and a taxonomy. 2000. Disponível em: < http://www.reusability.org/read/>. Acesso em: 15 mar. 2013. 